\title{
LA POLIFONÍA DE PLATÓN: DE LA UNIVERSIDAD A LA PLURIVERSIDAD REFORMA UNIVERSITARIA Y MODERNIZACIÓN ${ }^{1}$
}

\author{
NAPOLEÓN SALTOS
}

Facultad de Jurisprudencia Ciencias Políticas y Sociales Universidad Central del Ecuador

\footnotetext{
${ }^{1}$ Ponencia presentada en el Congreso "Universidad en el Camino de la Innovación Pedagógica”, realizado en Quito del 21 al 22 de noviembre de 2013, y organizado por el Instituto Universitario de Capacitación Pedagógica de la Universidad Central del Ecuador.
} 


\section{RESUMEN}

Hoy uno de los campos principales de disputa es el campo del conocimiento $y$, con ello, el campo de la universidad. La disputa entre un modelo monofónico, que gira en esferas en torno a un centro que piensa, vigila y controla. Una universidad jerarquizada, con centros encargados de la producción de conocimiento, ciencia, y con periferias prescritas a partir del orden generador. Y un modelo polifónico que traza la armonía en la diversidad, aún más, que busca pasar de la armonía de la convergencia. A partir de este dilema, la disputa sobre la universidad se mueve en tres campos principales: el rol de la universidad en las nuevas condiciones de la globalización de la sociedad del conocimiento; el nivel de democratización hacia abajo de la educación superior de calidad; y la constitución del sujeto del cambio universitario.

Palabras claves: Universidad, armonía monofónica, polifonía espacial, capitalismo, conocimiento, calidad, reforma.

\section{ABSTRACT}

Today one of the main areas of contention is the field of knowledge and thus, the field of college. The dispute between a mono model, spheres revolving around a center that thinks, monitors and controls. A hierarchical university with centers for the production of knowledge, science, and prescribed by the generator from peripheries. And a polyphonic model that traces the harmony in diversity, even more, looking to spend Harmony convergence. From this dilemma, the dispute over the university moves in three main areas: the role of the university in the new conditions of globalization of the knowledge society, the level of down democratization of higher education quality, and the constitution of the subject of university change.

Keywords: University, monophonic harmony, polyphony space, capitalism, knowledge, quality, reform. 


\section{¿Dónde iremos a buscar modelos? La América Española es original. Originales han de ser sus instituciones y su gobierno. \\ $Y$ originales los medios de fundar unas $y$ otro. \\ O inventamos o erramos. SIMÓN RODRÍGUEZ}

\section{POLIFONÍA}

La historia de Occidente quedó marcada por la visión aristotélica de la armonía monofónica: la perfección del círculo y de las esferas.

En La Caverna, Saramago (2000) retoma el mito presentado por Platón y traza la absorción del mundo del trabajo, de la artesanía y del campo por la maquinaria de la gran industria, de la metrópoli, del capital, y descubre el secreto de la producción en serie, no sólo de objetos, sino de seres humanos, que, atrapados en la caverna, ven la realidad únicamente por las sombras proyectadas en el muro. Tiene un desenlace optimista con la fuga de la familia Algor fuera de los límites de la ciudad y del mega-mall.

Hay otro texto de Platón sobre la polifonía espacial, "donde cada planeta es asistido por una Sirena, que canta un sonido una nota, y de todos ellos, los ocho, surge un acorde de una sola armonía" (Libro VII de La República en Ferris, 2012, p. 79).

Aristóteles señaló que esta teoría, "pese a la gracia y originalidad con que ha sido formulada, es sin embargo falsa" (En el cielo, en Ferris 2012, p. 80) y presentó una visión del mundo ordenado, monofónico, con esferas concéntricas en torno a la tierra. Tolomeo trazó la visión aristotélica.
Se requirieron 19 siglos para derribar esta visión en la astronomía, con el giro copernicano. Kepler estaba con Platón, partió de la concepción de que "Ios movimientos de los cielos no son más que una cierta polifonía (inteligible, no audible) eterna" (Las armonías en el Mundo, en Ferris, 2012, p. 81). Pasó de las órbitas regulares de las circunferencias y las esferas aristotélicas a descubrir que "la órbita de cada planeta describe una elipse, uno de cuyos focos ocupa el Sol". Y luego a la Segunda Ley, a la variación de la velocidad de los planetas conforme a la distancia del Sol, la relación entre tiempo y espacio (Ferris, 2012, p. 85).

El Renacimiento expresó el paso a la polifonía en diversos campos. La música se adelantó. Las fugas de Bach suplantaron "el canto llano y las melopeas de las catedrales medievales (...) por la polifonía, la música de muchas voces" (Ferris, 2012, p81). Y en el campo de las ciencias se abrió un diálogo con los descubrimientos matemáticos, médicos, astronómicos que venían desde Oriente.

Todavía por un tiempo nuestra América pudo ensayar la posibilidad de otra modernidad en torno al ethos barroco, (Echeverría, 2008) la superposición de las visiones de Occidente y de Oriente.

Pero finalmente se impuso la racionalidad de la modernidad capitalista: el imperio del valor de cambio sobre el valor de uso, de la cantidad sobre la calidad, de la uniformidad sobre la diversidad, Apolo sobre Dionisio. El eurocentrismo como la razón de la historia.

El fundamento a la modernidad capitalista está en la instauración sistémica de la división entre el trabajo manual y el trabajo intelectual, entre "los que saben" y "los que no saben", la privatización del saber, la 
vinculación del saber al poder. Una revolución educativa empieza por romper esta fractura, abrir un diálogo de saberes diferentes, de papeles diferentes en el saber. Una ruptura que implica la formación de intelectuales orgánicos contrahegemónicos, ubicados en el campo de la praxis, de la juntura entre teoría y práctica, del procesamiento de los saberes prácticos, de las experiencias y experimentaciones para elaborarlos teóricamente, por el cauce de la abstracción, y retornar al concreto de pensamiento como una totalidad compleja contigua a la realidad que se conoce.

La historia del eurocentrismo está marcada con sacrificios humanos a los dioses del capital, pero que salta una y otra vez a terrenos y cielos nuevos: la condena de la Inquisición a Galileo desplazó el salto de la ciencia hacia el Norte de Europa en donde la Reforma abrió nuevos campos de pensamiento. "La física del universo copernicano sería dilucidada por Isaac Newton, nacido en Woolsthorpe, Lincolnshire, el día de Navidad de 1642, el año de la muerte de Galileo" (Ferris, 2012, p. 110).

Ante la crisis civilizatoria de Occidente, el salto es hacia un diálogo de saberes entre los aportes de Occidente y los aportes de otras visiones civilizatorias; la superación del eurocentrismo no está en la negación de los aportes de Occidente, sino en su apertura al diálogo con los otros cauces civilizatorios; apertura que se opera por el lado de la potenciación y recuperación del hilo crítico acallado en cada etapa de la propia modernidad occidental por el poder dominante, por el saber oficial. En este nuevo mundo complejo, nuestra América se presenta como un campo de búsquedas alternativas.

El tiempo histórico se mueve en múltiples órbitas elíptica en espiral. El capital busca imponer su orden en torno a la reproducción y la acumulación ampliadas. Ha pasado de la soberanía territorial al biopoder, al disciplinamiento de los cuerpos y las personas y al control de las poblaciones. La forma final es la segurización del poder.

En tiempos de crisis estructural como los que vivimos, el capital "retorna" en espiral a las formas extremas: la depuración del valor de cambio de toda forma de valor de uso, para moverse en la autonomía especulativa del dinero y el capital financiero. El "retorno" a la acumulación originaria, a la "acumulación por desposesión": el capital rentista como soporte del capital financiero. Y en el campo de la política, la depuración del poder como dominación de toda forma de acuerdo, para moverse en la relación amigo-enemigo.

Un tiempo de paradojas. La historia abierta a nuevas polifonías y los arrebatos del capital para imponer su razón monofónica, a la fuerza. Y sin embargo una y otra vez salta la polifonía, la vida, las complejidades estructurales, las sociedades abigarradas, sobre todo allí en donde hay una mayor distancia, una desconexión, del centro.

\section{UNIVERSIDAD EN DISPUTA}

Hoy uno de los campos principales de disputa es el campo del conocimiento $y$, con ello, el campo de la universidad.

El universitario es un tipo peculiar de producción y enseñanza del saber que pertenece a lo mejor que trajo consigo la civilización moderna, (...) sobre todo por el hecho de que va siempre acompañado, de manera esencial, por un momento autocrítico. Es un saber que al mismo tiempo que contribuye a la modernidad es un crítico implacable de ella (Echeverría, 2009).

La disputa no es únicamente en torno a la producción del conocimiento, sino a una forma específica, el conocimiento y pen- 
samiento crítico. El conocimiento abarca diversos campos: ciencia, tecnologías, cultura, arte.

La disputa entre un modelo monofónico, que gira en esferas en torno a un centro que piensa, vigila y controla, en nombre del discurso de la excelencia y la calidad, con satélites dependientes, y apunta a una modernización funcional de la educación superior. Una universidad jerarquizada, con centros encargados de la producción de conocimiento, ciencia, y con periferias prescritas a partir del orden generador.

Y un modelo polifónico que traza la armonía en la diversidad, aún más, que busca pasar de la armonía de la convergencia, a la armonía de la disonancia, de la superposición de voces. Una especie de armonía de los sonidos de la calle y la plaza, en un diálogo entre la cultura alta y baja, que son reconstruidos en una nueva sensibilidad sin predominancias; o la superación de la centralidad de la propia armonía, para pasar al ritmo y a la intensidad. Y con ello empezar a descubrir nuevas formas de conocer y hacer ciencia, nuevos modelos de educación y pedagogía. El paso de la universidad a la pluriversidad, no tanto en la simple coexistencia de la primera fase del ethos barroco, sino en la construcción de diálogos de saberes, conocimientos, sabidurías para un nuevo cauce civilizatorio.

La disputa actual se da en el marco del debilitamiento de la fuerza utópica del iluminismo liberal, de la fuerza del saber y de la educación, para pasar a un tiempo de la "razón cínica" (Sloterdijk, 2004), la subordinación del saber al poder, a la voluntad: la actuación a pesar del saber, el predominio ya no sólo del ethos realista, sino del ethos pragmático.
Una disputa a tres bandas: la vieja universidad mercantilizada, la universidad modernizada y la universidad reformada.

Esta disputa parte de la presencia de "realidades ambivalentes, fundadas en el progreso técnico, (que) aparecen continuamente y reeditan el conflicto de lo innovador con lo obsoleto. (...) El enfrentamiento tan característico de la modernidad entre lo moderno y lo tradicional, encarnada en una lucha siempre renovada entre el partido de los "vándalos" y el de los humanistas." Esta contradicción está atravesada por "un conflicto que la modernidad tiene consigo misma o una inconsistencia o contradicción inherente a la modernidad misma: como si la modernidad establecida o "realmente existente" tratase de conciliar dos proyectos de sí misma, incompatibles el uno con el otro" (Echeverría, 2008).

El primer proyecto (...) acepta la doble incitación cuantitativa y cualitativa- de transformación civilizatoria y plantea toda una reconstrucción de la humanidad del ser humano en medio de la naturaleza. El segundo, interesado en mantener la estrategia civilizatoria del Occidente mercantil, acepta sólo la incitación cuantitativa de transformación y plantea una potenciación del dominio humano sobre la naturaleza, mediada por el modo de producción capitalista de la riqueza social" (Echeverría, 2008).

La historia de Occidente no es lineal. Hay una lucha permanente, a pesar del predominio de la forma capitalista se presenta un hilo subterráneo de visiones críticas en cada fase.

(E)n la modernidad "realmente existente" prevalece (...) una especie de destino ineluctable que condena a la primera de las dos posibilidades de modernización del mundo a estar sometida o subordinada bajo la otra. Se trata de un destino que condena, en primer lugar, a la subordinación de la capacidad política del sujeto social concreto o "natural" -preocupado por la dimensión cualitativa de sí mismo y del mundo de su vida- bajo la "voluntad cósica" de un pseudo-sujeto sustitutivo, el capital, con su obsesión productivista abstracta, puramente cuantitativa; capital que no es 
en verdad sino la objetivación enajenada y cosificada del mismo sujeto social. Es un destino que además, en segundo lugar, y como consecuencia de lo anterior, condena al "valor de uso" de los objetos del mundo de la vida a existir subordinado bajo el "valor de cambio" de los mismos, es decir, condena a la subsunción de la "forma natural" de los objetos de ese mundo bajo la "forma de valor" que tienen ellos mismos en la economía mercantil (un valor económico que está siempre, incesantemente, en proceso de autovalorizarse)" (Echeverría, 2008).

Esta contradicción pasa por diferentes ciclos. Actualmente los resultados de la "tercera revolución científico-técnica”, reactualizan la relación entre la naturaleza y la sociedad y, con ello, reactualizan la contradicción interna de la modernidad: o la continuidad de una modernidad dominada por la lógica del capital, bajo nuevas formas sometidas por el capital financiero y rentista; o una modernidad basada en el retorno del sujeto social emancipado y del sentido de la vida y del valor de uso.

A partir de este dilema, la disputa sobre la universidad se mueve en tres campos principales: el rol de la universidad en las nuevas condiciones de la globalización de la sociedad del conocimiento; el nivel de democratización hacia abajo de la educación superior de calidad; y la constitución del sujeto del cambio universitario.

\section{EL ROL DE LA UNIVERSIDAD}

La universidad vive actualmente un doble asedio tanto global como local: desde el mercado y desde el Estado, desde la apropiación privada del conocimiento y desde el control del poder sobre el conocimiento. Ya no se trata únicamente de la lucha ante el dominio del mercado del período neoliberal; sino que hay una nueva batalla ante el dominio de una modernización disciplinaria promovida desde arriba, desde el dominio del Estado
A partir del siglo XIX la universidad se presenta como el principal campo de producción y difusión del conocimiento, en particular, de la ciencia.

El asedio global se presenta bajo dos formas: la ruptura de la hegemonía de la universidad en la producción y difusión del conocimiento y en la formación de los productores de conocimiento y de los líderes de la sociedad. Y las nuevas formas de la división internacional del trabajo intelectual, que se expresa como jerarquización: universidades e instituciones de investigación, encargados de la producción legitimada de ciencia, conocimiento y tecnología, y universidades encargadas de su difusión y reproducción ampliada.

Esta no es una batalla local, es una batalla global, no se trata de una batalla de corta duración sino que viene desde atrás. Se dirige en contra de la función y la naturaleza misma de la Universidad.

La universidad ha perdido la exclusividad o la preeminencia en la producción del conocimiento y en la formación de los cuadros de la sociedad. La crisis de hegemonía (Santos, 2007) de la universidad implica que ya no es el único, ni el principal centro de producción de conocimiento y de formación; está asediada por los mass media, la opinión pública, el grupo de pares, los centros empresariales. En el caso de la universidad pública se añade la combinación del discurso de la ineficacia de lo público con el asedio de las universidades privadas de "calidad".

El triunfo del neoliberalismo significó una derrota estratégica de las fuerzas revolucionarias, y esta derrota impactó en la educación. "El desembarco invisible", bajo el mando del FMI, para "el cambio de paradigma en la educación pública desde una 
filosofía de derecho social y gratuito hacia una concepción mercado-céntrica", opera desde los años 80 (Báez, 2009).

Esta batalla está ligada al rediseño de la división del trabajo intelectual, bajo las nuevas condiciones del capital mundial.

La combinación de modelos de mercado y la extrañación del papel de la universidad por una deformación de la autonomía y la sobrepolitización, desembocó en una crisis profunda de la universidad ecuatoriana, que afectó no sólo a su funcionamiento interno, sino a la legitimidad ante la sociedad.

A este proceso René Báez denomina "el desembarco invisible", la funcionalización de la universidad ecuatoriana. La universidad es trazada bajo el paradigma de la empresa privada, la educación se convierte en una mercancía: "se trataría, en suma, de vincular la formación de nuestros profesionales conforme a las necesidades del capital mediante la homogeneización del proceso formativo y de sus "productos", facilitar su circulación como cualquier otro bien, abrir 'paquetes educativos', equiparar títulos y diplomas, etc." (Báez, 2009).

La mano invisible del mercado buscó construir la universidad a su imagen y semejanza: la universidad mercado-céntrica, la universidadempresa, encargada de producir mano de obra homogénea-intercambiable a nivel global, dotada de competencias, con calificación técnica suficiente para triunfar en el mercado global profesional. Introdujo la división internacional del trabajo intelectual entre las grandes universidades de los países centrales, con capacidad de producir conocimiento, ciencia y tecnología; y las universidades de las periferias, encargada de expandir la fórmula extraña en paquetes tecnológicos recubiertos de atrayentes envolturas de publicidad y marketing (Saltos, 2009b).
En los noventa llega un segundo "desembarco" mucho más sofisticado, bajo el timón del Banco Mundial y una red de universidades sobre todo europeas. Ya no se trata únicamente de la presión del mercado, sino de la articulación de la reforma educativa a la reforma del Estado, bajo una doble línea de paradigmas: la eficiencia y la meritocracia, de un lado, y la gobernanza y la participación ciudadana institucionalizada, de otro.

En el centro se coloca la reforma curricular: la igualdad y la equidad se convierten en homologación para la movilidad de carreras, estudiantes y docentes, el enfoque se centra en la educación por competencias, la traducción en el campo de la educación del concepto de la gobernanza.

La visión banco-mundialista y las diversas variantes de modernización "refleja" operadas en los países periféricos, se basan en la fractura final entre ciencia y tecnología, para poder diferenciar entre universidades del conocimiento y universidades de formación de profesionales. Aquí reside el punto clave para responder a la pregunta, Universidad, ¿para qué?

Empero, la universidad sigue siendo el centro de una forma especial de producción y enseñanza-aprendizaje de conocimiento y saberes: la vinculación con el pensamiento crítico, la proyección universal de los mismos. En los otros campos, particularmente en la ligazón con los mercados o los Estados, los objetivos inmediatos y los intereses instrumentales coartan las perspectivas de universalización y el poder de crítica del pensamiento.

Si se trata de privilegiar la "formación de profesionales de calidad", la solución es simple: la importación de modelos exito- 
sos, con alguna adaptación local, flamantes formas de neocolonialismo cultural.

Pero si el tema es "producir conocimiento bajo sus diferentes formas -ciencia, cultura, tecnología- y pensamiento crítico" y sobre esta base orientar la "formación de los estudiantes para la vida", la perspectiva es compleja: está ligada a la construcción de un proyecto de soberanía nacional y regional, desde el Sur, a la creación de condiciones para un diálogo intercultural entre lo más avanzado de la ciencia y la docencia de Occidente y los aportes desde la originalidad del Sur, desde la originalidad de nuestra América.

En la crisis de la universidad se refleja también la responsabilidad de las fuerzas que dominaron la vida estudiantil en las décadas de los setenta a los noventa, y en la ausencia de un proyecto alternativo capaz de disputar la educación superior en una perspectiva diferente.

La universidad se ubica en el cruce de la producción y difusión de la ciencia, y su organización (Wallerstein, 2006). La tendencia global hasta los setenta fue el disciplinamiento de las ciencias: la diferenciación de las "tres culturas", humanidades, ciencias naturales y ciencias sociales; y la demarcación de campos específicos de las disciplinas. Y sobre esta base la organización de universidades por facultades, con un variación posterior en torno a la organización por departamentos.

A partir de los setenta se abren dos direcciones contradictorias: la superposición de campos disciplinares y la especialización. Empezamos a pasar del "desencantamiento" del mundo a un nuevo "reencantamiento" (Prigogine y Stengers, 1983) desde el conocimiento. De una perspectiva de separación de las disciplinas empezamos a pasar a la articulación de las ciencias en campos transdisciplinarios y a la superación de las "tres culturas": un reencuentro de las ciencias naturales y las ciencias sociales, que implica superar la visión de la neutralidad de la ciencia y la introducción del tiempo y el espacio como categorías trasversales.

La batalla por la universidad se polariza entre el dominio del mercado y el poder del capital, frente a la búsqueda de enlazamientos de la educación con la liberación. De un lado, en el núcleo están las presiones por la modernización globalizadora, los rankings de las universidades, a partir de parámetros controlados por las redes de universidades e instituciones calificadoras, ubicadas en los países centrales. Y de otro, las búsquedas de nuevas formas de conocimiento y de educación: la pedagogía liberadora de Paulo Freire o la investigación-acción de Fals Borda.

América Latina ha vivido esta historia bajo formas reflejas, con momentos de recuperación de la identidad. La Reforma de Córdoba abre espacios de cambio a partir de una reorganización de la universidad, orientada a la producción de ciencia y conocimiento comprometidos con la sociedad. Los intentos de reforma han pasado por un enfrentamiento al poder: "Si en nombre del orden se nos quiere seguir burlando y embruteciendo, proclamamos bien alto el derecho sagrado a la insurrección" (Córdoba, 1918).

La reforma en nuestra América se mueve en una lucha anticolonialista, la lucha contra "el 'mimetismo intelectual' evidenciado en la repetición acrítica de modelos científicos con la finalidad de repetir o confirmar lo que se hace en otras latitudes, así como 
el riesgo a confundir "neutralidad valorativa" con objetividad científica" (Herrera, 2013).

En nuestro país, el intento más importante es la Segunda Reforma Universitaria, encabezada por Manuel Agustín Aguirre. La derrota de este intento abre las puertas a la mercantilización y decadencia de la universidad ecuatoriana.

\section{LA MODERNIZACIÓN REFLEJA}

Darcy Ribeiro (1978) distingue dos formas de modernización: la modernización refleja, funcional a las variaciones cíclicas del sistema; y una modernización con reformas, orientada a la liberación nacional y social. No está en discusión la necesidad del cambio y de la transformación estructural de la Universidad; el debate está sobre qué tipo de cambio.

Ante la "fuga neoliberal", surgió el consenso sobre la necesidad de un cambio profundo de la educación superior. Asistimos a una reorganización de la universidad: la necesidad de la modernización. La disputa entre la modernización funcional o la modernización con reforma.

El modelo oficial ha cambiado la normativa: Constitución, LOES, Reglamentos. Articula el sistema de educación superior al Plan de desarrollo y a la política gubernamental, en la perspectiva de la ciencia aplicada y la tecnología, en la reedición de un neopositivismo.

Ante la crisis de hegemonía, la respuesta oficial es la calificación y clasificación de las universidades (evaluación y acreditación): el discurso de la calidad.

Las jerarquías globales se replican en lo local: universidades de primera, de docencia e investigación, con capacidad de crea- ción de conocimiento, actúan como "tinktank", para la formación de cuadros de dirección; y universidades de segunda, de docencia, con capacidad de formación de profesionales y funcionarios. El eje está en la clasificación de las universidades:

- En la cúspide las universidades del conocimiento, administradas por el gobierno: el Proyecto Yachay, la "ciudad del conocimiento", con la Universidad de investigación en tecnología experimental, ${ }^{2}$ la Universidad Amazónica Ikiam, ${ }^{3}$ la Universidad Educativa, La Universidad de las Artes. A esta línea base se articulan el IAEN, convertido en la Universidad de postgrado del Estado, la ESPE que reúne a las instituciones universitarias de las Fuerzas Armadas,

\footnotetext{
2 "La Universidad de Investigación de Tecnología Experimental Yachay tiene como misión promover la investigación científica, la generación y difusión del conocimiento para resolver necesidades en un ambiente interdisciplinario, con el objeto de formar profesionales creativos e innovadores, de alta calidad humana, que contribuyan proactivamente al fortalecimiento de las capacidades sociales y al desarrollo del Ecuador y de la región." http://www.yachay.ec/universidad-deinvestigacion-cientifico-experimental/, consulta mayo de 2013.

3 Presentado como "uno de los proyectos más importantes, ambiciosos y estratégicos del Estado ecuatoriano, su creación e implementación en la región amazónica del país, como un centro de estudios superiores de excelencia y categoría mundial, es un paso fundamental hacia la construcción de la sociedad del conocimiento justa y solidaria que el Gobierno nacional plantea como su objetivo estratégico para apuntalar el desarrollo tecnológico y equitativo del Ecuador". Espinoza Augusto, Universidad Amazónica. Vida tierra conocimiento, http://www.conocimiento.gob.ec/ikiam/, consulta mayo de 2013.
} 
y la creación de un Instituto dirigido a la Policía.

Se trata de un modelo de modernización por enclave, ${ }^{4}$ orientado hacia un modelo bío-extractivista, en el que no participa la universidad pública. La misión de este primer nivel es la formación de científicos y gerentes de dirección de los organismos públicos, la constitución de "tink-tanks" de pensamiento articulados al desarrollo global.

El señalamiento de límites reales de las universidades, sobre todo en el campo de la investigación, se ha convertido en el justificativo para generar un modelo de enclave para las universidades gubernamentales, en particular para Yachay, no sólo en los planes curriculares, sino también en la composición del cuerpo docente e investigativo.

El proyecto Yachay es presentado como la piedra filosofal: "será un dispositivo para transformar la relación compleja existente entre el sistema universitario, el tejido empresarial, la diseminación del saber académico y científico, la incorporación de los profesionales recién egresados a la vida profesional y económica, y la transformación del tejido productivo; en última instancia, permitirá la modificación radical del modelo de acumulación y del modo de regulación de la economía ecuatoriana" (Yachay, 2013).

4 En nuestro país hay una disputa histórica entre la modernización de la educación por enclave, impulsada en su momento por García Moreno; o la reforma a partir de las condiciones propias de nuestra realidad y el aporte internacional: o creamos o erramos.
Para su crítica quizás hay que empezar por la recomendación de Terry Eagleton (2006):

No hay mejor punto de partida para la reflexión que la risa; hablando con mayor precisión, los espasmos del diafragma normalmente ofrecen mejores oportunidades para la reflexión que los espasmos del alma. (...) El chiste de la historia no es simplemente «el colapso de la representación de clases en un bufido de risa libertaria»; no, es bastante más, es el humor de la propia historia a expensas de una burguesía que sólo es capaz de disfrazar de esplendor épico su debilidad:

el discreto encanto de la burguesía, el discreto encanto de iniciar la historia para repetirla.

Este primer nivel se presenta como el modelo normativo de la reforma universitaria y cuenta con recursos abundantes. El modelo pedagógico se orienta a la vinculación con la ciencia, desde una visión neopositivista, y las tecnologías avanzadas sobre todo en los campos de la bío-tecnología, en articulación con el capital rentista mundial. $^{5}$

En este nivel habría que ubicar a las dos universidades internacionales de postgrado, la FLACSO-Sede Ecuador y

\footnotetext{
${ }^{5}$ David Murdock, el magnate de la DOLE: “Es importante tener un lugar para hacer cosas modernas y diferentes que no se han hecho antes, va a ser importante que el Ecuador sea un líder en la educación de los jóvenes en la región, porque ellos son el futuro del mundo. Es un proyecto espectacular (Yachay)", expresó. Del mismo modo indicó que apoyará en la transferencia de tecnología entre la Ciudad del Conocimiento y el Centro de Investigación Murdock. Tomadodehttp://www.elciudadano.gob.ec/index.php? opti-

on=com_content $\&$ view=article\&id=36891: multi millonario-de-eeuu-ofrece-ayuda-para-laconstruccion-de-yachay-ciudad-delconocimiento \& catid=40: actualidad\& Itemid $=63$, consulta abril 2013.
} 
la Universidad Andina Simón Bolívar, que se rigen con una normativa especial.

- Regulación de las universidades privadas, con condiciones de apertura. Hay que diferenciar dos niveles: las universidades clase A, tipo Universidad San Francisco, que replican modelos norteamericanos, junto a otras como las universidades Católicas, La UTPL, orientadas a la formación de cuadros gerenciales altos y medios, sobre todo para el sector privado. Y universidades de segundo nivel, orientadas a la formación de profesionales tecnólogos.

- Disciplinamiento y límites de la universidad pública. Hay que diferenciar dos niveles: las cinco universidades públicas con una mayor calificación, entre las cuales la Universidad Central del Ecuador es la más grande e importante; con la propuesta de una acreditación internacional, bajo parámetros y la guía de universidades internacionales, sobre todo norteamericanas; orientadas a la formación de profesionales. Y las universidades de segundo nivel, orientadas a la formación de tecnólogos y profesionales medios y bajos.

Esta clasificación está articulada a un redefinición territorial del Estado, con una distribución de las universidades del conocimiento, que jugarían el papel de ordenamiento de las otras universidades públicas y privadas, en los nodos internacionales; las universidades con mejor calificación en los nodos regionales; mientras los nodos locales contarían únicamente con universidades de educación continua o Institutos tecnológicos.

En lugar de una planificación para el conjunto del sistema de educación superior que permita potenciar la diversidad de identidades y la calidad de las universidades, para un aporte compartido al desarrollo e independencia de nuestro país y nues- tros pueblos, el modelo oficial impulsa un modelo homogeneizado con universidades que compiten por un rol clasificado jerárquicamente.

Ante la crisis de legitimidad, la respuesta oficial es la selección de los estudiantes y los docentes: el discurso de la meritocracia y el fetichismo de los títulos.

Ante la crisis institucional, la respuesta oficial es el paso al comando del Estado: el discurso de la autonomía responsable.

El modelo oficial de Alianza País, absorbe la energía del cambio y la orienta a una modernización en que predominan los movimientos reflejos desde los modelos importados con algunas tendencias de reformas: una especie de vía junker de modernización de la universidad, desde el control del Estado.

La presión desde arriba tiene un efecto positivo, pone en movimiento un sistema anquilosado, pero tiene un límite, la actuación por disciplinamiento y control; coloca en el centro la relación de la universidad con los planes de desarrollo, la producción y la tecnología.

El acto reflejo se mueve dentro de la tendencia sistémica del capital: la reproducción de la división internacional del trabajo intelectual a nivel interno, bajo la forma de la división social del trabajo intelectual. El sistema capitalista funciona sobre la base de la ruptura entre trabajo intelectual y trabajo material-manual, entre teoría y práctica. En el campo del conocimiento esta división parte de la discriminación entre los que saben y los que no saben, una nueva forma de iluminismo en tiempos de la razón cínica.

La historia de la universidad pública ecuatoriana ha estado marcada más por la forma "casona", en vínculo con la sociedad, 
que por la forma "claustro", encerrada en la producción de conocimiento, orientada a la producción y la tecnología. Esta tradición encarna una forma diferenciada de hacer ciencia y cultura en nuestra América: todavía la vinculación entre teoría y práctica, la constitución de la praxis como campo del conocimiento, la vinculación con lo social antes que con lo productivo.

Los cambios instituidos desde arriba desvalorizan estas raíces y privilegian las formas introducidas de la organización universitaria: sustituyen la reforma por la evaluación y la acreditación, centran las modificaciones en los cambios curriculares, en las políticas administrativas de la educación superior.

Dos dinámicas centrales: la rearticulación al nuevo orden mundial del conocimiento y el control estatal del sistema. La primera se expresa en la reproducción de la jerarquización de las universidades a nivel interno, con universidades del conocimiento y universidades de formación profesional. La segunda se expresa en un modelo de enclave, organizado en torno al discurso de la "calidad" de la educación, mientras se abandona la pertinencia; un modelo selectivo que tiende a la exclusión.

El proyecto Yachay, convertido en el proyecto emblemático de la Revolución Ciudadana, encarna estas tendencias:

El modelo de universidad apunta hacia la implantación de un estándar universitario de carácter instrumental, productivista, de corte empresarial, funcional al mercado y a las políticas de crecimiento económico. Esta tendencia vendría acompañada por una creciente opacidad de los límites del conocimiento como un bien público o como un producto capitalizado al servicio de actividades de lucro. En otras palabras, el sistema universitario (...) estaría avanzando hacia una suerte de capitalismo académico que implicaría una revisión de la misión misma de la universidad y de su relación con su entorno social" (Villavicencio, 2013).

En todo caso, hay que ver esta batalla, no sólo como un riesgo, sino más bien como una oportunidad: la presión del Estado sobre el Sistema de Educación Superior obliga a las universidades a un proceso de cambio.

Hay dos peligros: responder desde el sometimiento y la obediencia, renunciando a lo que constituye a la universidad como tal, pensar críticamente; o responder desde una perspectiva gremialista de "defensa de las conquistas". No hay posibilidad de defensa hacia atrás, es el tiempo de que la comunidad universitaria se ponga a la cabeza de los cambios profundos que requiere la universidad: una posición crítica y autocrítica, con alternativas de cara a un proyecto de soberanía y liberación local y latinoamericana.

El primer paso es devolver al debate sobre la universidad un piso propio, a partir de nuestras condiciones y nuestra identidad, reubicar el cambio desde las potencialidades de la universidad ecuatoriana y latinoamericana y no sólo desde la crítica negativa, pasar desde una visión de competición y actuación aislada a la proyección de un sistema basado en la colaboración y la complementación.

Una perspectiva alternativa parte de una relectura de nuestra historia y de revinculación con las luchas por la reforma universitaria en nuestro Continente, con los imaginarios de Córdoba en Argentina, de Mella en Cuba y de Manuel Agustín Aguirre en Ecuador. La "creación heroica" no se refiere sólo a la copia externa, sino también a la repetición dogmática del propio pasado.

\section{LA DEMOCRATIZACIÓN Y EL SUJETO}

La universidad debe resolver, 
"la contradicción entre la jerarquización de los saberes especializados, por un lado, a través de las restricciones del acceso y certificación de las competencias, y por otro lado, por las exigencias sociales y políticas de la democratización de la universidad y la reivindicación de igualdad de oportunidades para los hijos de las clases populares" (Santos, 2007).

Se ha roto el consenso ante la necesidad de la selección y calificación de saberes y aprendizajes y el objetivo de la democratización y universalización del derecho a la educación superior; y se ha producido una crisis de legitimidad de las universidades como instituciones con un tratamiento especial.

A ello se suma una crisis administrativa que se presenta como la contradicción entre la reivindicación de autonomía de parte de las instituciones de educación superior y la presión para someter la institución a criterios de eficiencia y productividad empresarial y social, o para colocar como techo de la acción universitaria la ligazón al Plan de desarrollo de un régimen. La autonomía es asediada por el control del mercado y del Estado.

La proliferación de universidades-galpón y una visión masista de la democratización de la Universidad terminaron por hacer estallar el consenso en torno al libre ingreso construido en base al imaginario de la Reforma de Córdoba.

La visión neoliberal impulsa modelos de selectividad excluyentes, tanto en el ingreso como en el egreso: la jerarquización de universidades se extiende a la jerarquización de docentes y estudiantes, bajo el paradigma de la meritocracia.

Las respuestas ante esta contradicción se mueven en dos ámbitos: el sistema de salida y entrada a la universidad; y las for- mas de participación, o la definición del sujeto.

Las respuestas han pasado por ciclos alternativos de apertura y exclusión. En nuestro país la lucha estudiantil por el libre ingreso de los años sesenta inauguró un largo período de democratización del acceso (input) a la educación superior. Empero esta orientación desembocó en una masificación perjudicial para la universidad, con bajos niveles de salida (output) de estudiantes graduados y alto nivel de deserción. El modelo actual apunta a formas excluyentes ligadas al objetivo de la calidad.

El reto atraviesa todo el proceso universitario: empieza por construir una alternativa que articule la democratización de la educación superior, con sistemas de capacitación que equiparen las oportunidades para el acceso a la Universidad. Transformar el proceso de enseñanza-aprendizaje, a partir de la reconstitución de la comunidad universitaria (docentes-estudiantestrabajadores) como sujeto protagónico del proceso pedagógico y del impulso de un modelo pedagógico que fortaleza la participación activa y crítica de los actores del proceso de enseñanza-aprendizaje. Garantizar un perfil de salida que prepare al estudiante para la vida en una sociedad compleja.

La relegitimación parte de la recuperación de producir conocimiento y saberes, parte de la autoridad académica y científica, como base para recuperar la voz universitaria ante los problema fundamentales de la sociedad y la humanidad.

El segundo aspecto tiene que ver con la autonomía universitaria. En nuestra América, a raíz de la Reforma de Córdoba la autonomía se convirtió en un constitutivo de 
la universidad. A partir de los sesenta en nuestro país se instituye un sistema autónomo de educación superior, aunque la dependencia financiera y las intervenciones del poder, con clausuras incluidas, ponían los límites.

Este sistema entra en crisis cuando se lee en clave neoliberal: autonomía para el mercado libre de la mercancía educativa, sin rendición de cuentas ni a la sociedad ni al Estado, a pesar de los lemas de "luchar junto al pueblo".

Si en el período neoliberal el asedio a la autonomía se dio por el cerco presupuestario, en la actualidad se da por el control disciplinario de los organismos rectores del sistema, dependientes del régimen.

Anteriormente la autonomía era una característica constitutiva de la universidad; ahora se trata de una capacidad dependiente de la correlación de fuerzas entre las universidades frente al mercado y al Estado.

La tendencia actual es al fortalecimiento del control estatal de las universidades. Dos dispositivos centrales operan: el control del presupuesto y los sistemas de evaluación y acreditación.

Las reformas normativas desde la Constitución de Montecristi y, sobre todo la LOES, desplazan el sujeto al control de Estado, o más precisamente de régimen. Los dos organismos rectores, el CES y el CEAACES son externos al sistema de educación superior y operan bajo formas de control. No se ha logrado crear canales de diálogo con poder de decisión compartida. Si bien el Estado, o más bien el régimen, recupera la capacidad de definir líneas políticas para la educación superior, se instaura un línea más bien vertical y centralizada. La autonomía universitaria queda reducida al inte- rior de cada Universidad, mientras se anula respecto al funcionamiento del sistema. En la modernización desde arriba, se suplanta la reforma con la evaluación y el control.

El reto es redefinir la autonomía "responsable" ante el Estado y ante la sociedad, a partir de la defensa y unidad de la educación superior pública. "Para salir de su posición defensiva, la universidad debe estar segura que la reforma no se hace en su contra" (Santos, 2007). Y para ello el punto de partida es recuperar la iniciativa del cambio desde la comunidad universitaria, para redefinir la relación con la sociedad y el Estado, la redefinición de la autonomía no sólo de cada universidad al interior, sino del sistema en su conjunto.

Hay una larga tradición de lucha en este sentido en nuestra América. La Rebelión de Córdoba es ante todo un grito de democratización:

Fundar la garantía de una paz fecunda en el artículo conminatorio de un reglamento o de un estatuto es, en todo caso, amparar un régimen cuartelario, pero no una labor de ciencia. Mantener la actual relación de gobernantes a gobernados es agitar el fermento de futuros trastornos. Las almas de los jóvenes deben ser movidas por fuerzas espirituales. Los gastados resortes de la autoridad que emana de la fuerza no se avienen con lo que reclaman el sentimiento y el concepto moderno de las universidades (Córdoba, 1918).

El sujeto universitario se construye en la definición de una identidad en torno al modelo educativo, en la organización de una fuerza creativa, en el impulso de planes y estrategias que apunten a la recuperación de la hegemonía, la legitimidad y la autonomía de las universidades y del sistema de educación superior a partir de su capacidad de producción de conocimiento, ciencia y pensamiento crítico y de la for- 
mación de los cuadros de dirección de la sociedad y el Estado. El punto de partida es la reconstitución de un gran acuerdo de política universitaria dentro de la comunidad integrada por docentes, estudiantes y trabajadores.

El protagonismo de los estudiantes es decisivo, si se busca una reforma desde abajo. Recuperar el sentido de Córdoba:

La juventud ya no pide, exige que se le reconozca el derecho a exteriorizar ese pensamiento propio en los cuerpos universitarios por medio de sus representantes. Está cansada de soportar a los tiranos. Si ha sido capaz de realizar una revolución en las conciencias, no puede desconocérsele la capacidad de intervenir en el gobierno de su propia casa (Córdoba, 1918).

\section{¿ES PRESCINDIBLE LA UNIVERSIDAD?}

Cuando hablamos de universidad, hablamos ante todo de producción, difusión y enseñanza-aprendizaje de conocimiento $y$ saberes, de valores y actitudes de vida. Las políticas neoliberales en la educación superior se asientan en una fractura entre ciencias y humanidades.

Es sólo en la perspectiva del predominio de la política económica sobre la política republicana (...) que aquello que caracteriza esencialmente el saber universitario, esto es, la unidad dialéctica entre las ciencias y las humanidades, parece carecer de sentido y en la que esta últimas y su vocación crítica se presentan como estorbosas y prescindibles para la verdadera ciencia que sería la ciencia matematizadora (Echeverría, 2009).

Un neopositivismo duro que termina por reducir la ciencia a la racionalidad instrumental de la razón del capital.

La importación de modelos externos basados en el fetichismo del positivismo y de los títulos pone en riesgo la fuerza trans- formadora de los procesos latinoamericanos.

Y en esta perspectiva América Latina corre el riesgo de renunciar a su propia fuerza. En nuestro Continente la forma de hacer ciencia es diferente a la del Norte: aún no se ha producido una ruptura total entre ciencia y humanidades, entre teoría y práctica; y eso permite una mirada más holística. La universidad latinoamericana puede aportar, no sólo en su defensa, sino como alternativa ante la crisis del sistema capitalista, una forma de conocimiento que supere el largo camino de fractura del conocimiento, puede escribir la otra historia.

Nacida en los estertores del Medioevo, "en el debate entre teología y filosofía, entre fe y razón, la historia de la universidad se construye en forma paralela a la historia de la ciencia y en el campo de la relación saber-poder. Una historia de fisuras y separaciones, de especializaciones y reducciones." Primero la separación de la teología y la filosofía, luego la separación de la filosofía y las ciencias. "La filosofía y las humanidades son catalogadas como no-ciencias, un conocimiento inferior, hasta convertirse en superfluas frente a la razón instrumental." Luego, "dentro de las ciencias se traza una nueva frontera entre ciencias físicas (naturales) y las ciencias sociales. (...) El resultado es la constitución de tres culturas del saber: las ciencias naturales - "duras", exactas" -, las ciencias sociales y las humanidades como disciplinas separadas." Las universidades se organizan paralelamente en base a este patrón: el modelo napoleónico de las facultades (Saltos, 2009b).

En el capitalismo tardío llegamos a una nueva ruptura entre ciencia y tecnología; la propia ciencia cae víctima de la utilidad, para dar paso a la proclamación de la supremacía de las tecnologías, y reducir la educación a la adquisición 
de tecnologías adecuadas, las competencias, para el éxito profesional (Saltos, 2009b).

Pero éste no es un camino lineal. En forma paralela se ha escrito la otra historia que hoy se presenta como la exigencia de la reintegración de la ciencia y como un diálogo civilizatorio de saberes. $Y$ se presenta como gérmenes y signos de las luchas antisistémicas de los pueblos.

La Universidad en Latinoamérica es necesaria sólo en la perspectiva de la relación entre ciencias y humanidades, en la perspectiva del poder del pensamiento crítico que es su fundamento. La Universidad pública, en particular, es necesaria sólo si entra a disputar la producción de conocimiento y la formación de los líderes de la sociedad y del Estado; si recupera su carácter creativo.

La alternativa pasa por volver a conectar los tiempos de la reforma en nuestro continente: los tiempos de Córdoba en el nacimiento del Estado nacional-liberal, los intentos truncos de la Segunda Reforma en nuestro país, el esclarecimiento y desmarcamiento de campos con la modernización actual. El reto es salir de los muros formales de la excelencia o la calificación, para desatar las fuerzas de la creación y la crítica. Pero allí el poder del orden se vuelve la nueva frontera.

América Latina es a la vez extremo Occidente y originalidad que proviene desde la raíz de los pueblos originarios, indígenas y afros. El camino de la reforma universitaria pasa por un modelo barroco que abra el cauce de un diálogo fructífero entre las formas occidentales de conocimiento $y$ saberes y los aportes de la originalidad latinoamericana. Parafraseando a José Carlos Mariátegui, también la universidad latinoamericana "no es calco ni copia, sino creación heroica".

\section{BIBLIOGRAFÍA}

BÁEZ, René (2009). El desembarco invisible, en Saltos, 2009.

CÓRDOBA (1918). Manifiesto liminar, disponible en:

http://www.unc.edu.ar/institucional/historia/r eforma/manifiesto, consulta noviembre 2013.

EAGLETON, Terry (2006). La estética como ideología, Madrid, Trotta.

ECHEVERRÍA, Bolívar (2008). El ethos barroco y los indios, Revista de Filosofía "Sophia", QuitoEcuador. № 2/, http://es.scribd.com/doc/22551904/BolivarEcheverria-El-Ethos-Barroco, consulta noviembre de 2013.

ECHEVERRÍA, Bolívar (2009). ¿Es prescindible la Universidad?, en SALTOS, 2009.

FERRIS, Timthy (2012). La aventura del espacio. De Aristóteles a la Teoría de los cuantos: una historia sin fin, Barcelona, Crítica.

HERRERA, Nicolás (2013). Orlando Fals-Borda, pedagogo de la praxis, Ponencia presentada en el Congreso "Universidad en el camino de la innovación pedagógica", realizado en Quito del 21 al 22 de noviembre de 2013 y organizado por el Instituto Universitario de Capacitación Pedagógica de la Universidad Central del Ecuador.

PRIGOGINE, Ilya e Isabelle STENGERS (1983), La nueva alianza, Alianza Editorial.

RIBEIRO Darcy (1978), El proceso civilizatorio. Etapas de evolución socio-cultural.

Proyecto Yachay (2013). www.yachay.ec/universidad-deexperimentacion-cientifico-experimental/, consulta noviembre de 2013. 
SALTOS, Napoleón (compilador) (2009). Reforma universitaria hoy, Quito, Universidad Central del Ecuador.

SALTOS Napoleón, (2009b). La otra historia, en SALTOS 2009.

SANTOS Boaventura De Souza (2007). La Universidad en el Siglo XXI, Para una reforma democrática y emancipatoria de la universidad, Cides-Umsa, Asdi y Plural editores, La Paz, disponible en http://www.boaventuradesousasantos.pt/medi a/universidad_siglo_xxi-.pdf, consulta noviembre 2013.

SARAMAGO, José (2000). La caverna, Alfaguara, Madrid.

SLOTERDIJK, Peter (2004). Crítica de la razón cínica, Siruela, Madrid.

VILLAVICENCIO, Arturo (2013). De la Universidad funcional a la Universidad de la razón, Quito, Universidad Andina.

WALLERSTEIN, Inmanuel (Coordinador), Comisión Gubelkian para la reestructuración de las ciencias sociales, 2006, Abrir las ciencias sociales, México, Siglo XXI, 9a edición, disponible en http://www.slideshare.net/Gatojazzy/inmanuel -wallerstein-abrir-las-ciencias-sociales, consulta noviembre de 2013.

YACHAY (2013), www.yachay.ec/universidadde-experimentacion-cientifico-experimental/, consulta noviembre de 2013. 